always grossly overestimate the correlation about which the VIH makes a prediction.

It might be argued that unreliability has reduced the correlations reported to the low values they show, but reliabilities would need to be very low indeed, near .25 at most, to raise the values reported even as high as .5 , let alone to the values of .8 and .9 , on which Glanzer and Clark (1962, 1963 , 1964) base their conclusions. A supplementary study showed that the correlation between written and spoken description lengths, the two being obtained one week apart, is 59 , which suggests that reliability for written descriptions is reasonably high.

The results reported here show that there is little or no support for the VLH when statistical artifacts are removed from the picture and pictorial patterns are put in.

\section{REFERENCES}

GLANZER, M., \& CLARK, W. H. Accuracy of perceptual recall: An analysis of organization. Journal of Verbal Learning \& Verbal Behavior, 1962, 1, 289-299.

GLANZER, M., \& CLARK, W. H. The verbal-loop hypothesis: Binary numbers. Journal of Verbal Learning \& Verbal Behavior, $1963,2,301-309$.

GLANZER, M., \& CLARK, W. H. The verbal-loop hypothesis: Conventional figures. American Journal of Psychology, 1964, 77, 621-626.

ROSS, J. The verbal-loop hypothesis: A statistical audit. Australian Journal of Psychology, 1968, 20, 233-235.

\title{
An explanation of the effect of tilt on the Poggendorff illusion
}

\section{A. W. PRESSEY, University of Manitoba, Winnipeg 19, Canada}

An expalanation of the interaction between the effect of tilt and orientation on the Poggendorff illusion is given in terms of three facts: (1) that the illusion decreases with a decrease in distance between the parallel lines, (2) that the illusion increases as the angle of the oblique decreases, and (3) that the retinal image changes systematically as the target is tilted backwards.

In 1966, Leibowitz and Toffey measured the Poggendorff illusion as a joint function of orientation and tilt of the target. Although tilt was found to interact with orientation, they did not offer any explanation of their results. The present report is an attempt to account for Leibowitz and Toffey's findings on the basis of three well known facts. The first is that the Poggendorff illusion increases as the angle that the interrupted oblique forms with the parallel line decreases. The second fact is that the Poggendorff illusion decreases as the distance between the parallel lines decreases. And the third is that systematic changes in the size of the retinal image occur as a target is tilted backwards. The general rule is that vertical distances decrease, but horizontal distances remain constant.

The four orientations of the Poggendorff illusion used by Leibowitz and Toffey are shown in Fig. 1 ( $A$ to $D$ ). In addition, $3 \mathrm{deg}$ of tilt $(0,55$, and 80$)$ were employed in a factorial design. The results showed that tilt had no effect on either Fig. $1 \mathrm{~A}$ or $1 \mathrm{D}$, but the illusion decreased with increase of tilt in Fig. 1C. On the other hand, the Poggendorff illusion increased as Fig. 1B was tilted backwards.

Now consider the changes that occur in the retinal image as Fig. 1 $\mathrm{A}$ is tilted. The distance between the parallel lines remains the same, and the distance defining the angles of the oblique (e in Fig. 1A) also remains constant because both of these are horizontal distances. Therefore, the illusion should not change as the target is tilted. In Fig. 1D, tilting the target backwards results in a decrease of the distance between the two parallel lines that produces a decrease in illusion. However, the distance $\mathrm{h}$, which defines the angle of the oblique, decreases (the angle becomes more acute), and this results in an increase in illusion. Therefore, as the target is tilted, there are two contradictory trends, which probably cancel each other out to produce no effect.

Tilting Fig. $1 B$ results in the distance $f$ being reduced, which produces a more acute angle. The distance $j$, however, remains the same. Therefore, the illusion increases with an increase in tilt. Finally, tilting Fig. 1C decreases the distance k, but the angle remains constant. Hence, the illusion decreases with an increase in tilt. It will be noted that all the predictions derived from the changes in the retinal image that are consequent upon tilt are substantiated by the results reported by Leibowitz and Toffey.

\section{REFERENCE}

LEIBOWITZ, H., \& TOFFEY, S. The effect of rotation and tilt on the magnitude of the Poggendorff illusion. Vision Research, 1966, 6, 101-103.
A

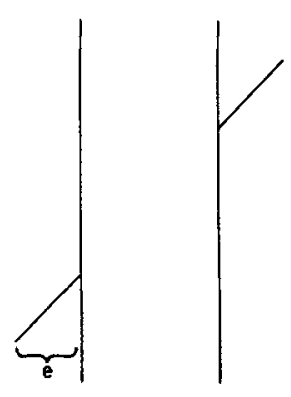

B

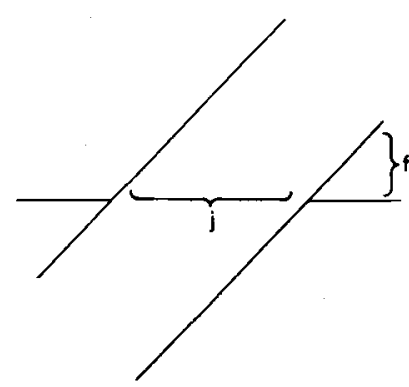

C

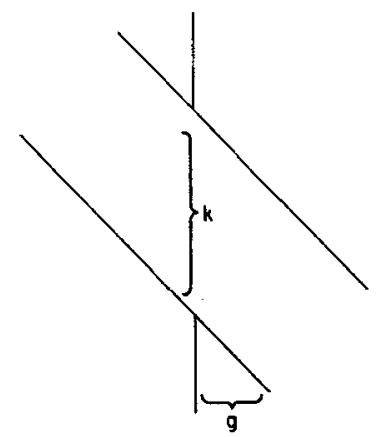

D

Fig. 1. Orientations of the Poggendorff illusion employed by Leibowitz \& Toffey (1966). 